\title{
Dissociation between CSF total tau and tau protein phosphorylated at threonine 231 in Creutzfeldt-Jakob disease
}

\author{
Katharina Buerger ${ }^{\mathrm{a}, *}$, Markus Otto ${ }^{\mathrm{b}, 1}$, Stefan J. Teipel ${ }^{\mathrm{a}}$, Raymond Zinkowski ${ }^{\mathrm{c}, 2}$, \\ Kaj Blennow ${ }^{\mathrm{d}, 3}$, John DeBernardis ${ }^{\mathrm{c}}$, Daniel Kerkman ${ }^{\mathrm{c}}$, Johannes Schröder ${ }^{\mathrm{e}, 4}$, \\ Peter Schönknecht ${ }^{\mathrm{e}}$, Lukas Cepek ${ }^{\mathrm{b}}$, Cheryl McCulloch ${ }^{\mathrm{c}}$, Hans-Jürgen Möller ${ }^{\mathrm{a}}$, \\ Jens Wiltfang ${ }^{\mathrm{f}, 5}$, Hans Kretzschmar ${ }^{\mathrm{g}, 6}$, Harald Hampel ${ }^{\mathrm{a}}$ \\ a Dementia Research Section and Memory Clinic, Alzheimer Memorial Center and Geriatric Psychiatry Branch, Department of Psychiatry, \\ Ludwig-Maximilian University, Nussbaumstrasse 7, 80336 Munich, Germany \\ ${ }^{\mathrm{b}}$ Department of Neurology, University of Goettingen, Robert-Koch Str. 40, 37075 Goettingen, Germany \\ ${ }^{c}$ Applied NeuroSolutions Inc. (formerly Molecular Geriatrics Corp.), 50 Lakeview Pkwy, Vernon Hills, IL 60061, USA \\ d Department of Clinical Neuroscience, Unit of Neurochemistry, University of Göteborg, Sahlgren's University Hospital, Mölndal, Sweden \\ e Section of Gerontopsychiatry, University of Heidelberg, Voss-Str. 4, D-69115 Heidelberg, Germany \\ ${ }^{\mathrm{f}}$ Department of Psychiatry, Friedrich-Alexander University Erlangen-Nurnberg, Schwabachanlage 6, 91054 Erlangen, Germany \\ ${ }^{\mathrm{g}}$ Institute of Neuropathology, Ludwig-Maximilian University Munich, Feodor Lynen Strasse 23, 81377 München, Germany
}

Received 13 July 2004; received in revised form 26 August 2004; accepted 1 December 2004

\begin{abstract}
To study the potential diagnostic value of abnormally phosphorylated tau protein in Creutzfeldt-Jakob disease (CJD) compared to Alzheimer's disease (AD), we determined levels of tau phosphorylated at threonine 231 (p-tau ${ }_{231}$ ) and of total tau (t-tau) in cerebrospinal fluid (CSF) of CJD patients, AD patients, and healthy controls (HC). CJD patients showed excessively high t-tau levels but relatively low p-tau 231 concentrations compared to the AD group. $t$-tau alone yielded the best diagnostic accuracy to differentiate between CJD and AD patients, when compared to $\mathrm{p}-\mathrm{tau}_{231}$ and the $\mathrm{p}$-tau $\mathrm{ta}_{231} / \mathrm{t}$-tau ratio $(97,78$, and $95 \%$ correctly allocated cases, respectively). Our findings indicate a dissociation in the direction of change in CSF levels of t-tau and p-tau 231 in CJD when compared to AD.
\end{abstract}

(C) 2005 Elsevier Inc. All rights reserved.

Keywords: Creutzfeldt-Jakob disease (CJD); Alzheimer's disease (AD); Diagnosis; Differential diagnosis; Cerebrospinal fluid (CSF); Biological marker; Tau protein; Phosphorylated tau protein

Abbreviations: CJD, Creutzfeldt-Jakob disease; AD, Alzheimer's disease; CSF, cerebrospinal fluid; t-tau, total tau protein; p-tau 231 , tau protein phosphorylated at threonine 231; HC, healthy control; ELISA, enzyme linked immunosorbent assay; ROC, receiver operating characteristics; AUC, area under the curve; CAC, correctly allocated cases; MMSE, Mini Mental State Examination; M-W- $U$, Mann-Whitney- $U$-test

* Corresponding author. Tel.: +49 895160 5824; fax: +49 8951605865.

E-mailaddresses: katharina.buerger@med.uni-muenchen.de, hampel@med.uni-muenchen.de (K. Buerger), motto@gwdg.de (M. Otto), zinkowski@appns.com (R. Zinkowski), kaj.blennow@neuro.gu.se (K. Blennow), johannes_schroeder@med.uni-heidelberg.de (J. Schröder), jens.wiltfang@psych.imed.uni-erlangen.de (J. Wiltfang), hans.kretzschmar@med.uni-muenchen.de (H. Kretzschmar).

1 Tel.: +4955139 12905; fax: +495513914449.

2 Tel.: +1 847573 8025; fax: +1 8475738030 .

3 Tel.: +46 313431 791; fax: +46313432426.

4 Tel.: +49 6221 564403; fax: +49 6221562753 .

5 Tel.: +49 913185 36896/34160; fax: +49 913185 36002/36381.

6 Tel.: +49 89218078000 ; fax: +49 89218078037 . 


\section{Introduction}

Major efforts are under way to define biological markers of neurodegenerative disorders like Creutzfeldt-Jakob (CJD) and Alzheimer's disease (AD). Moreover, a biomarker to support differential diagnosis between CJD and AD would be clinically meaningful. In cerebrospinal fluid (CSF), determination of total tau protein ( $\mathrm{t}$-tau) concentration is of high diagnostic value for CJD with sensitivity and specificity levels above 90\% when distinguishing CJD from non-demented and demented neurological controls and $\mathrm{AD}[11,15,20,21]$. T-tau levels are elevated both in CJD and $\mathrm{AD}$ relative to controls, but the increase is several magnitudes higher in CJD compared to AD. Extreme concentrations of $t$-tau in the CSF of CJD patients most likely reflect the high acuity and magnitude of neuronal destruction.

In $\mathrm{AD}$, abnormal phosphorylation of tau protein is associated with the development of neurofibrillary tangles (NFT), a major hallmark of AD neuropathology which is usually absent in sporadic CJD. Abnormally phosphorylated tau protein (p-tau) can be detected in vivo in the CSF. Using monoclonal antibodies specific for different phosphorylated epitopes of tau, enzyme-linked immunosorbent assays (ELISA) have been developed that sensitively measure concentrations of CSF p-tau. Highly significant increases in CSF p-tau in AD patients have recently been demonstrated in independent studies, mainly using three different immunoassays specific for the phosphorylated epitopes threonine $231\left(\mathrm{p}-\mathrm{tau}_{231}\right)$ [12], threonine $181\left(\mathrm{p}-\operatorname{tau}_{181}\right)$ [22], and serine $199\left(\mathrm{p}-\mathrm{tau}_{199}\right)$ [10]. Evidence from these studies indicates that quantification of tau phosphorylated at these specific sites may improve early detection (as a prognostic and diagnostic marker) [2], differential diagnosis (as a classificatory marker) [3,4,7,18], and tracking of disease progression in $\mathrm{AD}$ (for review see $[1,8])$. Therefore, CSF measurement of $\mathrm{p}$-tau proteins comes closest to fulfilling criteria of a core feasible biomarker of $\mathrm{AD}[5]$.

In a recent study, a subgroup of patients with sporadic CJD showed high CSF levels of tau protein abnormally phosphorylated at threonine 181 (p-tau 181$)$ [20]. Measurement of CSF p-tau 181 , however, did not improve diagnostic power between CJD and AD compared to measurement of t-tau [20]. Another study showed an overlap of CSF p-tau 181 concentrations between $\mathrm{AD}$ and CJD patients and suggested the $\mathrm{p}$-tau $\mathrm{tan}_{181} / \mathrm{t}$-tau ratio as a potential biomarker to differentiate CJD from $\mathrm{AD}$ [16].

Phosphorylation of tau protein at threonine 231 (p-tau 231 ) has been hypothesized to be specific for AD [23]. To further explore the diagnostic value of phosphorylated tau protein in CJD and $\mathrm{AD}$, we investigated p-tau 231 in the CSF using a recently developed assay [12]. We hypothesized that in contrast to $\mathrm{AD}, \mathrm{CJD}$ patients would present a dissociation between increased levels of t-tau indicating a high rate of general neuronal loss, and relatively low or normal levels of p-tau 231 .

\section{Materials and methods}

CSF was obtained from 21 patients with CJD (12 women; age $64.2 \pm 8.4$ years). All patients were seen by a member of the national CJD surveillance team. Since June 1993, suspected cases of CJD in Germany have been reported to the CJD surveillance unit at the Department of Neurology at Georg-August-University in Göttingen. Each patient was visited by a research physician and examined using a standardized protocol. According to the clinical criteria, all suspected cases of CJD were classified as "probable", "possible", or "other" cases [15]. Before clinical staging into "probable" or "possible", all patients underwent at least one cranial CT or MRI, or both, to exclude ischemic stroke, hemorrhage, or space-occupying lesions as a cause of the illness.

The diagnostic criteria for the clinical CJD staging are as follows:

- Probable: patients with a rapidly progressive dementia of less than 2 years duration, periodic sharp-wave complexes in the EEG, and two of the following:

o Myoclonus.

o Visual or cerebellar symptoms.

o Pyramidal or extrapyramidal signs.

o Akinetic mutism.

- Possible: patients fulfilling the preceding criteria but without typical EEG abnormalities.

- Other: patients not fulfilling the criteria for possible or probable CJD.

- Definite: cases with immunohistochemical detection of the $\mathrm{PrP}^{\mathrm{SC}}$ in brain tissue [13].

Twelve CJD patients were later neuropathologically verified as definite CJD patients (10 sporadic and 2 familial); 9 were classified as probable CJD.

Further, we enrolled 37 patients with AD (35 probable; 2 possible AD according to NINCDS-ADRDA criteria [14]; 25 women; age $67.3 \pm 9.1$ years) and 10 healthy controls ( 8 women; age $67.7 \pm 7.7$ years). The groups were age and gender matched.

The protocol was approved by the local ethics committees and the Institutional Review Boards of the participating centers.

CSF sampling and processing was performed as described previously [7]. In detail, lumbar punctures were done between 9 and $11 \mathrm{a} . \mathrm{m}$. according to a routine protocol. CSF was collected in polypropylene tubes on ice in $0.5 \mathrm{ml}$ aliquots. In case of contamination with blood due to the procedure, the sanguineous CSF was collected in a separate tube. For scientific purposes, only clear CSF was used. For this study, a total of $1 \mathrm{ml}$ was taken. Aliquots were centrifuged at $4{ }^{\circ} \mathrm{C}$ at $10.000 \times g$ for $10 \mathrm{~min}$ and stored at $-80^{\circ} \mathrm{C}$ until analysis.

p-tau was measured by a newly developed enzyme-linked immunosorbent assay (ELISA) [12]. For total tau, we used a commercially available ELISA (Innogenetics, Belgium). 
Differences in age between groups were assessed with the Mann-Whitney-U-test. Group differences in the gender distribution were tested through the $\chi^{2-}$ test. Since sample sizes are less than $N=100$, we tested for normal distribution with the Kolmogorov-Smirnov-test. Tau proteins were normally distributed in the AD and HC groups, but not in CJD patients. Therefore, we used non-parametric approaches in our statistical analyses. Differences in mean CSF levels of the t-tau and p-tau 231 between groups were assessed with the Kruskal-Wallis test. Pairwise comparisons between groups were performed with the Mann-Whitney-U-test. Correlation between t-tau and p-tau 231 was assessed with Spearman's rank correlation. For t-tau, p-tau 231 , and the $\mathrm{p}$-tau $231 / \mathrm{t}$-tau ratio, sensitivity and specificity levels and numbers of correctly allocated cases were derived from receiver operating characteristic (ROC) curve analysis when the sum of specificity and sensitivity was maximized. Areas under the ROC curves (AUC) as measures of diagnostic accuracy were compared between t-tau, p-tau 231 , and the p-tau $231 / \mathrm{t}$-tau ratio using the algorithm of Hanley [9].

\section{Results}

Mean CSF p-tau 231 levels in CJD were $324.9 \pm 345.8$ $\mathrm{pg} / \mathrm{ml}, 613.6 \pm 337.5 \mathrm{pg} / \mathrm{ml}$ in $\mathrm{AD}$, and $92.9 \pm 104.8 \mathrm{pg} / \mathrm{ml}$ in $\mathrm{HC}$ (Fig. 1A). Levels of CSF total tau were $9406.6 \pm 7796.7 \mathrm{pg} / \mathrm{ml}$ in CJD, $609.0 \pm 258.8 \mathrm{pg} / \mathrm{ml}$ in $\mathrm{AD}$, and $340.9 \pm 117.7 \mathrm{pg} / \mathrm{ml}$ in HC (Fig. 1B). Only in the $\mathrm{AD}$ group, t-tau and p-tau 231 were correlated (Spearman's rho 0.627, $p<0.001$ ), but not in the CJD group (Spearman's rho $-0.118, p=0.61$ ), and the HC subjects (Spearman's rho
$0.018, p=0.96$ ) (Fig. 2A-C). The lack of correlation between tau proteins in the CJD group remained stable after exclusion of five CJD patients with extremely high t-tau concentrations (Spearman's rho $-0.147, p=0.59$ ). CSF p-tau 231 as well as t-tau levels were significantly different between groups $(p<0.001) . \mathrm{p}-\mathrm{tau}_{231}$ levels were higher in $\mathrm{AD}$ versus CJD $(p<0.01)$, AD versus HC $(p<0.001)$, and in CJD versus HC $(p<0.01)$. CSF t-tau was increased in CJD versus AD $(p<0.001), \mathrm{CJD}$ versus HC $(p<0.001)$, and in AD versus HC $(p<0.01)$.

The $\mathrm{p}$-tau $231 / \mathrm{t}$-tau ratio was significantly different between groups $(p<0.001)$ and lowest in patients with CJD, followed by controls and AD patients (CJD versus $\mathrm{HC}: p<0.01$; CJD versus $\mathrm{AD}: p<0.001$; $\mathrm{HC}$ versus $\mathrm{AD}: p<0.001)$. Results remained stable after exclusion of five CJD patients with extremely high t-tau values $(p<0.001$ over all groups; CJD versus HC: $p=0.012$; CJD versus AD: $p<0.001$; HC versus $\mathrm{AD}: p<0.001)$.

Discriminative power between CJD and AD was highest for t-tau alone with a sensitivity of 90\%, a specificity of $100 \%$, and $97 \%$ correctly allocated cases. Using the p-tau $231 /$ t-tau ratio, sensitivity was $91 \%$ at a specificity of $97 \%$ and $95 \%$ correctly allocated cases. CSF p-tau 231 yielded a sensitivity of $81 \%$, a specificity of $76 \%$ and correctly allocated $78 \%$ of cases. The area under the ROC curve was 0.75 for $\mathrm{p}$-tau $231,0.99$ for $\mathrm{t}$-tau, and 0.93 for the $\mathrm{p}$ $\operatorname{tau}_{231} / \mathrm{t}$-tau ratio (Fig. 3A-C). Comparison of AUCs yielded significant differences between $\mathrm{p}-\mathrm{tau}_{231}$ compared to t-tau $(p<0.001)$ as well as compared to the $\mathrm{p}$-tau $231 / \mathrm{t}$-tau ratio $(p<0.001)$ with lower values for $\mathrm{p}-\mathrm{tau}_{231}$. Between $\mathrm{t}$-tau and the $\mathrm{p}$-tau $231 / \mathrm{t}$-tau ratio, AUC was not significantly different $(p=0.99)$.
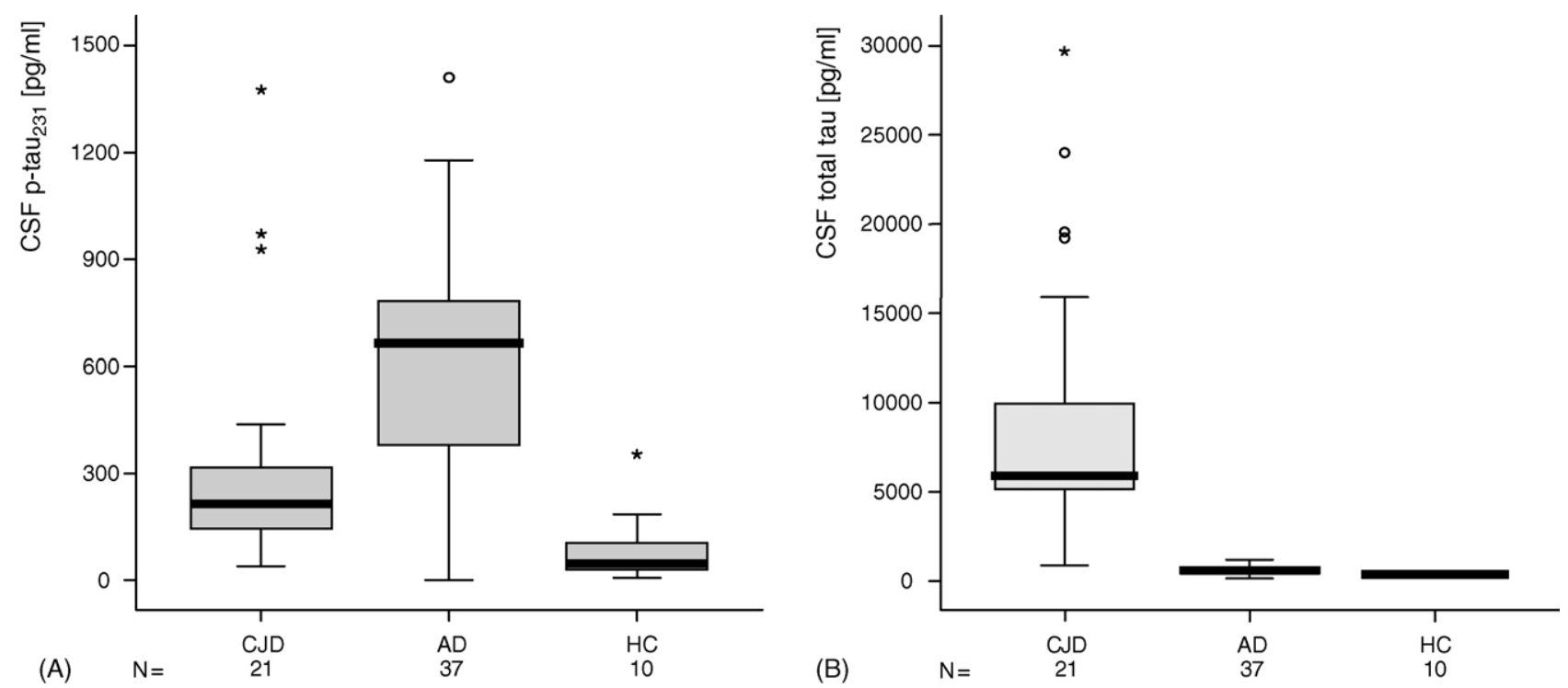

Fig. 1. (A) Boxplots of CSF p-tau 231 levels in CJD, AD, and HC subjects. (B) Boxplots of CSF t-tau levels in CJD, AD, and HC subjects. Boxes represent the median, the 25 th and the 75 th percentiles, bars indicate the range of data distribution. Circles represent outliers (values more than 1.5 box-length from the 75 th/25th percentile). The asterisks represent extreme values (value more than three box-length from the 75 th/25th percentile). 

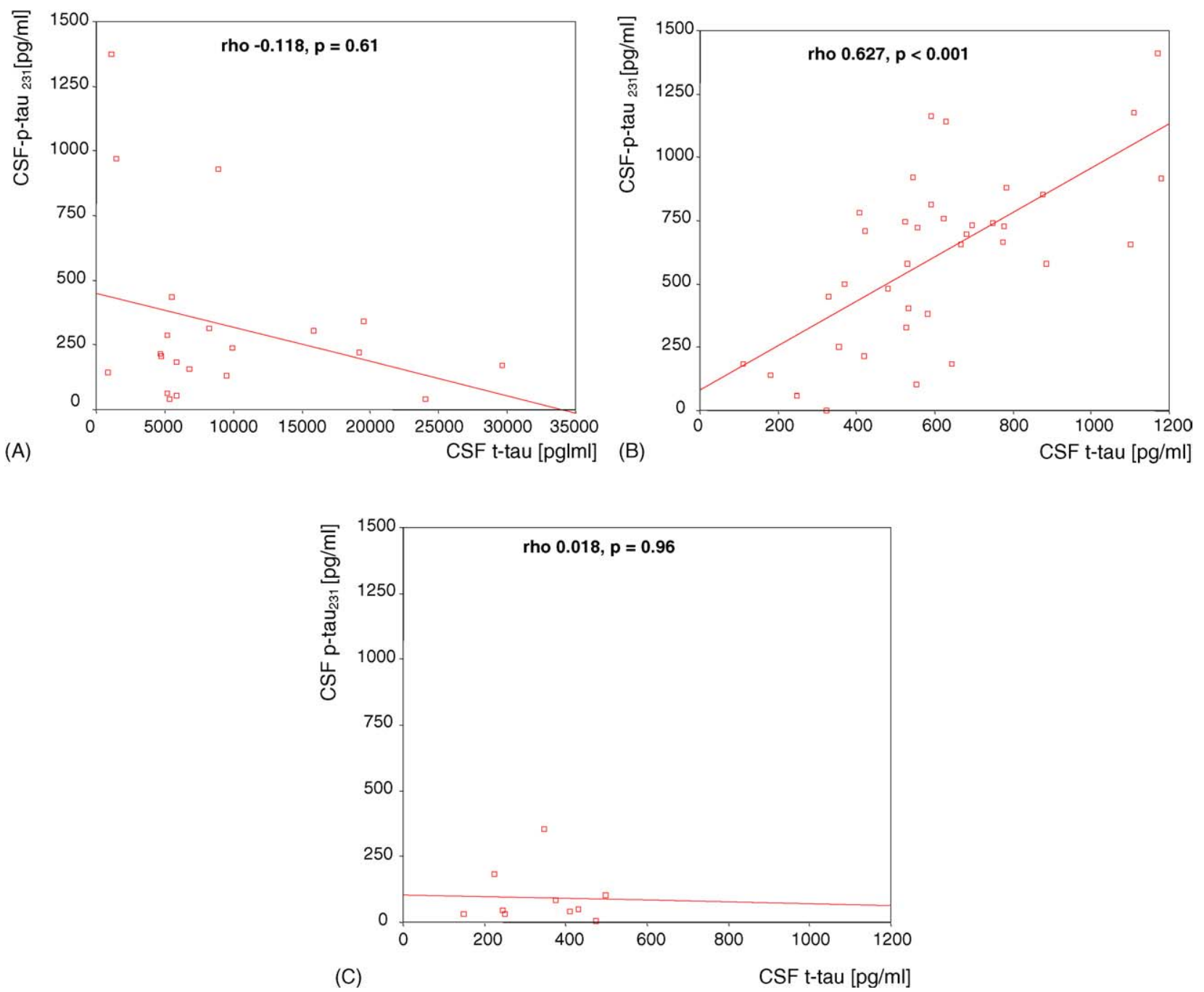

Fig. 2. (A) Correlation of CSF p-tau 231 and t-tau in CJD patients. (B) Correlation of CSF p-tau 231 and t-tau in AD patients. (C) Correlation of CSF p-tau 231 and t-tau in $\mathrm{HC}$ subjects.

\section{Discussion}

Our group studied p-tau 231 and t-tau in the CSF of patients with CJD, in AD patients, and in healthy controls. In agreement with earlier studies, we found excessively high levels of t-tau in CJD patients compared to the AD and the age-matched HC groups [15,20,21]. CSF p-tau 231 concentrations, however, were significantly lower in CJD patients compared to the $\mathrm{AD}$ group, indicating an opposite direction of change in $\mathrm{t}$-tau and p-tau 231 expression in the CSF of CJD patients.

The small number of controls enrolled in our study limits power to preclude a type II error, i.e. to assume that there is no difference between groups despite a true difference of markers in the population. Our results, however, showing significantly lower levels of CSF p-tau 231 and t-tau in HC subjects compared to AD patients as well as extremely high t-tau values in CJD compared to other diagnostic entities are in accordance with the literature $[2,3,4,7,11,15]$. The main findings of our study refer to the CJD and the AD groups, i.e. a dissociation between CSF p-tau 231 and t-tau in CJD patients and the diagnostic accuracy to differentiate CJD from $\mathrm{AD}$ using t-tau, $\mathrm{p}$-tau 231 , and the $\mathrm{p}$-tau $231 / \mathrm{t}$-tau ratio. Therefore, the relatively small $\mathrm{HC}$ group enrolled in the study is unlikely to affect the main findings of the study.

We found no improvement of diagnostic accuracy for the differentiation between CJD and AD patients through the p$\mathrm{tau}_{231} / \mathrm{t}$-tau ratio compared to $\mathrm{t}$-tau alone. In a recent study, the ratio of tau protein phosphorylated at threonine 181 and t-tau had been suggested as a useful measurement to identify patients with CJD [16]. In agreement with that study, we found that the p-tau/t-tau ratio was lowest in the CJD group compared to the other groups studied. The previously reported results, however, did not include an estimate of the diagnostic accuracy of p-tau, t-tau, or the p-tau/t-tau ratio.

We found significant differences in CSF p-tau 231 levels between the groups enrolled in our study. Van Everbroeck et al. [20] did not report a difference in CSF p-tau 231 between 


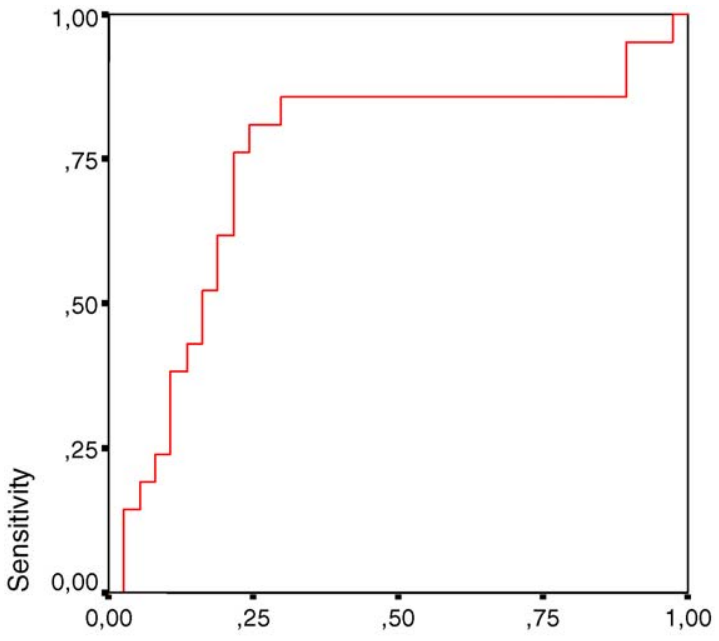

(A) 1 - Specificity

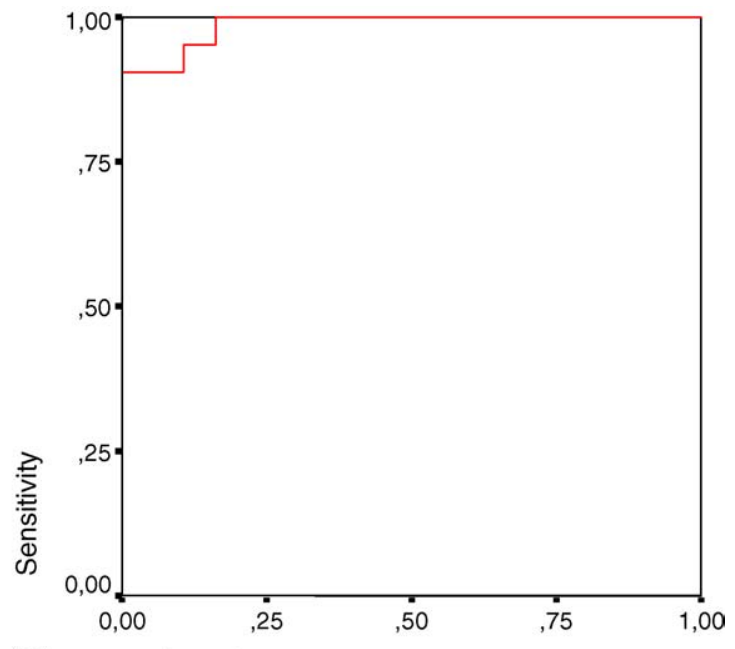

(B) $\quad 1$ - Specificity

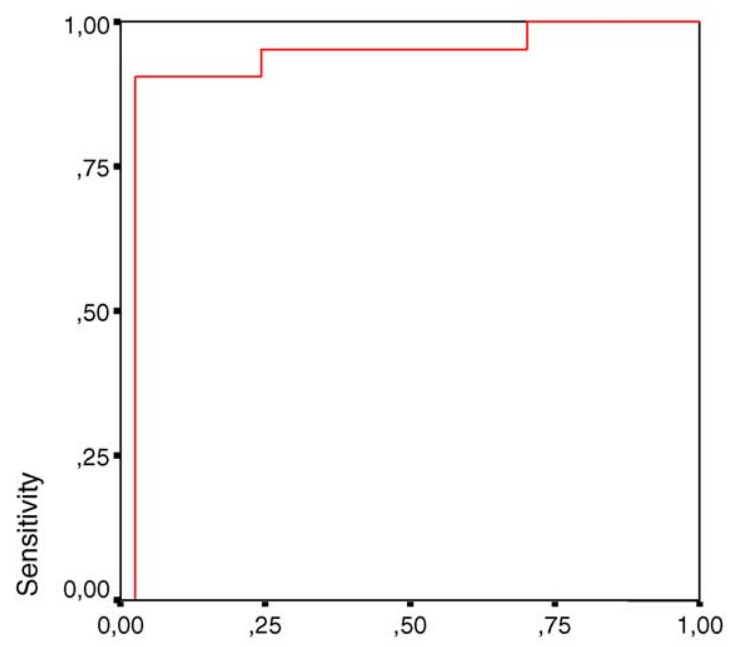

(C) 1 - Specificity

Fig. 3. Receiver operating characteristics curves (ROC) for CSF p-tau 231 (A), CSF t-tau (B), and the p-tau $231 / t$-tau ratio (C) when CJD patients were compared to the $\mathrm{AD}$ group.

CJD and AD patients, as well as HC subjects. This finding might be due to differences between the methods used (Everbroeck et al. determined CSF $\mathrm{p}$-tau 231 by western blot using polyclonal rabbit antibodies).

The elevation of CSF p-tau 231 in some of our CJD patients compared to HC subjects may not be merely an effect of neuronal damage and increased release of intracellular proteins, since there is no correlation between $\mathrm{t}$-tau reflecting neuronal damage and p-tau 231 in CJD. Increased CSF p-tau 231 in CJD might partly be due to underlying AD pathology and could partly account for an elevation of CSF p-tau 231 in our CJD patients compared to the $\mathrm{HC}$ subjects. Twenty-three percent of our CJD patients had high p-tau 231 levels whereas in the age between 60 and 69 , a prevalence of $\mathrm{AD}$ of only $0.3 \%$ has been reported [17]. Coexisting AD pathology has been described in CJD. In an Austrian neuropathological longitudinal study of 110 CJD patients, coexisting AD pathological changes have been described in $11 \%$ of cases of which $20 \%$ showed
NFT pathology [6]. Another study reported NFT pathology in 19\% of 65 sporadic CJD patients [19]. An increase of p-tau 181 in a subgroup of CJD patients, however, was not related to coexisting $\mathrm{AD}$ neuropathological features [20]. The increase of p-tau 231 in CJD might partly be due to induced (extra)cellular phosphorylation of tau protein or release of phosphorylated tau protein into the CSF in a subgroup of CJD patients. The role of differences in phosphorylation in CJD therefore should be addressed in future studies.

\section{Acknowledgements}

This work was supported by grants from the VolkswagenFoundation, Hannover, Germany (H.H.); the Hirnliga, e.V. (H.H., K.B.); from the Medical Faculty of the LudwigMaximilian University, Munich (K.B., S.J.T., H.H.); from the German Competency Network on Dementias 
(Kompetenznetz Demenzen), funded by the Bundesministerium für Bildung und Forschung (BMBF), Germany; and from the German Ministry of Health (grant number GZ: 325-4471-02/33, M.O., Sigrid Poser). The authors thank S. Poser, I. Zerr, B. Ciesielyck, M. Bodemer for scientific support, F. Jancu, B. Riemenschneider, and O. Pogarell, M.D., for clinical support, and T. Nolde, and H. Ott for technical assistance. We further thank Michael Ewers, Ph.D., for helpful discussion of the manuscript.

\section{References}

[1] Blennow K, Hampel H. CSF markers for incipient Alzheimer's disease. Rev Lancet Neurol 2003;2(10):605-13.

[2] Buerger K, Teipel SJ, Zinkowski R, Blennow K, Arai H, Engel R, et al. CSF tau protein phosphorylated at threonine 231 correlates with cognitive decline in MCI subjects. Neurology 2002;59(4):627-9.

[3] Buerger K, Zinkowski R, Teipel SJ, Arai H, DeBernardis J, Kerkman D, et al. Differentiation of geriatric major depression from Alzheimer's disease with CSF tau protein phosphorylated at threonine 231. Am J Psychiatry 2003;160:376-9.

[4] Buerger K, Zinkowski R, Teipel SJ, Tapiola T, Arai H, Blennow K, et al. Differential diagnosis of Alzheimer's disease with CSF tau protein phosphorylated at threonine 231. Arch Neurol 2002;59(8):126772.

[5] Frank RA, Galasko D, Hampel H, Hardy J, De Leon M, Mehta DP, et al. Biological markers for therapeutic trials in Alzheimer's disease. Proceedings of the biological markers working group; NIA initiative on neuroimaging in Alzheimer's disease. Rev Neurobiol Aging 2003;24(4):521-36.

[6] Hainfellner JA, Wanschitz J, Jellinger K, Liberski PP, Gullotta F, Budka H. Coexistence of Alzheimer-type neuropathology in Creutzfeldt-Jakob disease. Acta Neuropathol 1998;96(2):11622.

[7] Hampel H, Buerger K, Zinkowski R, Teipel SJ, Goernitz A, Andreasen $\mathrm{N}$, et al. Measurement of phosphorylated tau epitopes in the differential diagnosis of Alzheimer disease: a comparative cerebrospinal fluid study. Arch Gen Psychiatry 2004;61(1):95-102.

[8] Hampel H, Goernitz A, Buerger K. Advances in the development of biomarkers for Alzheimer's disease: from CSF total tau and Abeta (1-42) proteins to phosphorylated tau protein. Rev Brain Res Bull 2003;61(3):243-53.

[9] Hanley JA, McNeil BJ. A method of comparing the areas under receiver operating characteristics curves derived from the same cases. Radiology 1983;148:839-43.

[10] Itoh N, Arai H, Urakami K, Ishiguro K, Ohno H, Hampel H, et al. Large-scale, multicenter study of cerebrospinal fluid tau protein phosphorylated at serine 199 for the antemortem diagnosis of Alzheimer's disease. Ann Neurol 2001;50(2):150-6.
[11] Kapaki E, Kilidireas K, Paraskevas GP, Michalopoulou M, Patsouris E. Highly increased CSF tau protein and decreased beta-amyloid (142) in sporadic CJD: a discrimination from Alzheimer's disease? J Neurol Neurosurg Psychiatry 2001;71(3):401-3.

[12] Kohnken R, Buerger K, Zinkowski R, Miller C, Kerkman D, DeBernardis J, et al. Detection of tau phosphorylated at threonine 231 in cerebrospinal fluid of Alzheimer's disease patients. Neurosci Lett 2000;287:187-90.

[13] Kretzschmar HA, Ironside JW, DeArmond SJ, Tateishi J. Diagnostic criteria for Creutzfeldt-Jakob disease. Arch Neurol 1996;53(9):913-20.

[14] McKhann G, Drachman D, Folstein M, Katzman R, Price D, Stadlan EM. Clinical diagnosis of Alzheimer's disease: report of the NINCDS-ADRDA Work Group under the auspices of the Department of Health and Human Services Task Force on Alzheimer's disease. Neurology 1984;34:939-44.

[15] Otto M, Wiltfang J, Cepek L, Neumann M, Mollenhauer B, Steinacker $P$, et al. Tau protein and 14-3-3 protein in the differential diagnosis of Creutzfeldt-Jakob disease. Neurology 2002;58(2):192-7.

[16] Riemenschneider M, Wagenpfeil S, Vanderstichele H, Otto M, Wiltfang J, Kretzschmar $\mathrm{H}$, et al. Phospho-tau/total tau ratio in cerebrospinal fluid discriminates Creutzfeldt-Jakob disease from other dementias. Mol Psychiatry 2003;8(3):343-7.

[17] Rocca WA, Hofman A, Brayne C, Breteler MM, Clarke M, Copeland JR, et al. Frequency and distribution of Alzheimer's disease in Europe: a collaborative study of 1990 prevalence findings. The EURODEM-Prevalence Research Group. Ann Neurol 1991;30(3):381-90.

[18] Schoenknecht P, Pantel J, Hunt A, Wolkamm M, Buerger K, Hampel $\mathrm{H}$, et al. Levels of total tau and tau protein phosphorylated at threonine 181 in patients with incipient and manifest Alzheimer's disease. Neurosci Lett 2003;339:172-4.

[19] Van Everbroeck B, Pals P, Dziedzic T, Dom R, Godfraind C, Sciot R, et al. Retrospective study of Creutzfeldt-Jakob disease in Belgium: neuropathological findings. Acta Neuropathol 2000;99(4):358-64.

[20] Van Everbroeck B, Green AJ, Vanmechelen E, Vanderstichele H, Pals P, Sanchez-Valle R, et al. Phosphorylated tau in cerebrospinal fluid as a marker for Creutzfeldt-Jakob disease. J Neurol Neurosurg Psychiatry 2002;73(1):79-81.

[21] Van Everbroeck B, Quoilin S, Boons J, Martin JJ, Cras P. A prospective study of CSF markers in 250 patients with possible Creutzfeldt-Jakob disease. J Neurol Neurosurg Psychiatry 2003;74(9):1210-4.

[22] Vanmechelen E, Vanderstichele H, Davidsson P, Van Kerschaver E, Van Der Perre B, Sjogren M, et al. Quantification of tau phosphorylated at threonine 181 in human cerebrospinal fluid: a sandwich ELISA with a synthetic phosphopeptide for standardization. Neurosci Lett 2000;285(1):49-52.

[23] Vincent I, Zheng JH, Dickson DW, Kress Y, Davies P. Mitotic phosphoepitopes precede paired helical filaments in Alzheimer's disease. Neurobiol Aging 1998;19(4):287-96. 